\title{
Estudio sobre el Cumplimiento de las Asignaturas Ingeniería de Requisitos y Gerencia Informática con Respecto a la Guía Base de Conocimientos en Ingeniería de Software $\left(\right.$ Swebok $^{\odot}$ )
}

\author{
Andrea C. Alarcón-Aldana, Aida N. Martínez-Camacho y Mauro Callejas-Cuervo \\ Facultad de Ingeniería, Escuela de Ingeniería de Sistemas y Computación, Grupo de Investigación en \\ Software GIS, Universidad Pedagógica y Tecnológica de Colombia, Tunja, Boyacá - Colombia \\ (e-mail: acalarcon@gmail.com; nataly.martinez.17@hotmail.com; maurocallejas@gmail.com)
}

Recibido Ene. 14, 2016; Aceptado Mar. 8, 2016; Versión final May. 26, 2016, Publicado Oct. 2016

\begin{abstract}
Resumen
Se presenta un estudio realizado en los cursos de Ingeniería de Sistemas y Computación de la Universidad Pedagógica y Tecnológica de Colombia, sobre el estado actual de las asignaturas Ingeniería de Requisitos y Gerencia Informática con respecto a los contenidos y estrategias contenidas en la Guía Base de Conocimientos en Ingeniería de Software (Swebok@). El propósito de esta investigación es dotar al comité de currículo del Programa académico de un documento de revisión y estado actual de esas asignaturas con el propósito de realizar los ajustes necesarios y así propender por la mejora académica del mismo. Para el desarrollo de la investigación se realizó una serie de encuestas a distintos actores del contexto académico y mapeos a las asignaturas, identificando las estrategias pedagógicas y metodológicas usadas actualmente. Los principales resultados muestran que el cumplimiento de los contenidos de las asignaturas frente a la guía Swebok en Ingeniería de Requisitos es del 72.8\% y en la asignatura Gerencia Informática es $49 \%$.
\end{abstract}

\section{Study on the Compliance of the Courses Requirements Engineering and Information Technology Management with respect to the Software Engineering Body of Knowledge (Swebok@)}

\begin{abstract}
A study that was carried out in the Systems and Computer Engineering programme of the Universidad Pedagógica y Tecnológica de Colombia, on the current state of the subjects of the area of Software Engineering and Information Technology Management with respect to the contents and strategies included in the Software Engineering Body of Knowledge (Swebok@). The purpose of this research is to provide the Curriculum Committee of the academic programme with a revision document and the current state of these courses with the objective of making the necessary adjustments and thus fostering the academic improvement of the programme. A number of surveys were carried out on different actors within the academic context as well as mapping of the subjects, identifying the pedagogical and methodological strategies currently used. The main results show that the compliance of the subjects with the contents from the Swebok guide in Software Engineering is $72.8 \%$ and the subject Information Technology Management is $49 \%$.
\end{abstract}

Keywords. Swebok guide; requirements engineering; it management; bloom's taxonomy 


\section{INTRODUCCIÓN}

La naturaleza cambiante de la ingeniería del software, la generación de nuevos conceptos, técnicas y teorías de cómo planear, analizar, diseñar, construir y probar software además del incremento de la demanda de ingenieros, hace pertinente la formación integral de perfiles profesionales (Osuna y Luna, 2011), en los que la enseñanza debe ir a la par con estas actualizaciones, sin desconocer sus bases fundamentales, incluidas en diversos documentos guía, entre estos Software Engineering Body Of Knowledge (Swebok). Esta es una guía propuesta y aprobada por el Instituto de Ingenieros eléctricos y Electrónicos (IEEE Computer Society), la Organización Internacional de Estandarización (ISO), la Comisión Electrotécnica Internacional (IEC) y otros, con el fin de reunir y definir los conocimientos mínimos de la disciplina de Ingeniería del Software. Estos criterios están estructurados en diez áreas fundamentales en la versión del 2004: Requisitos de software, diseño, construcción, pruebas, mantenimiento, gestión de la configuración, gestión de la ingeniería de software, proceso de la ingeniería de software, herramientas y métodos de la ingeniería de software y calidad de software (Abran et al., 2004); y en la versión 3.0 de 2014, además de las diez mencionadas, se adicionan otras cinco áreas de conocimiento: Práctica profesional de la Ingeniería de software, Economía en la ingeniería de software, fundamentos de computación, Fundamentos matemáticos y Fundamentos en Ingeniería (Bourque y Fairley, 2014).

Swebok a través del tiempo se ha caracterizado por su versatilidad, ya que ésta guía ha sido usada como base para múltiples trabajos e investigaciones encaminados a la mejora de la enseñanza, como en el caso Siemens Corporate Development Center India donde se adaptó a la industria de requerimientos con resultados positivos en la formación de ingenieros (Samarthyam et al., 2012), también se realizó una integración de la seguridad al ciclo de vida de la Ingeniería del Software (Talib et al., 2010), así como también, esta guía ha sido base fundamental para la mejora del currículo de programas de Ingeniería del Software, Ingeniería de sistemas, Ingeniería informática y afines en diversas instituciones de educación superior (Alarifi et al., 2016; Kakeshita, 2015; Offutt, 2015; Quezadaa et al., 2015; Ramakrishnan, 2007). Es importante mencionar que en un entorno académico no solamente deben medirse los contenidos temáticos de un currículo sino además la parte psicológica, habilidades, competencias, ética profesional y conducta de los involucrados.

Para ello existen proyectos basados en Swebok como SWEBOS (Sedelmaier y Landes, 2015), y GSwE2009 (Ardis et al., 2011) que exploran el complemento del conocimiento, con base en lo anterior, en esta investigación se analizó el estado actual de las áreas Ingeniería de Requisitos (IEEE, 2011) y Gerencia Informática o gestión de la ingeniería de software (Nokes, 2007; PMI, 2016) en el programa Ingeniería de Sistemas y Computación de la Universidad Pedagógica y Tecnológica de Colombia (UPTC), frente a los contenidos plasmados en las áreas inherentes contempladas en la Guía Swebok, así como también se revisó qué estrategias pedagógicas se han utilizado y cuáles son las sugeridas de acuerdo a la taxonomía de Bloom; los anterior con el propósito de ofrecer un instrumento que apoye las actividades del comité curricular del programa para que realice los ajustes pertinentes en pro de la mejora en los procesos de enseñanza-aprendizaje.

El artículo se estructura de la siguiente manera, en primera instancia se describe la metodología empleada para desarrollar la investigación planteada, luego se presenta los resultados obtenidos de la aplicación de los instrumentos (mapeo y entrevistas); posteriormente se plantea el modelo pedagógico y metodológico adecuado para el programa de Ingeniería de Sistemas y Computación de la Universidad Pedagógica y Tecnológica de Colombia, y finalmente se exponen las conclusiones de la investigación.

\section{METODOLOGÍA}

Para el desarrollo de la investigación se implementó una metodología de tres fases, dirigidas por los objetivos planteados, como se describe a continuación.

\section{Fase I. Recolección de Información}

La primera fase se orienta al estudio de las temáticas planteadas por la guía Swebok en Requisitos de software y Gestión de la Ingeniería de software, y los parámetros de diseño necesarios para la definición de los contenidos que se ajusten a las temáticas definidas y en un contexto pedagógico basado en la Taxonomía de Bloom expresa en la versión 2004 de la guía, como una clasificación de los objetivos cognoscitivos de la educación, en cumplimiento del dominio del conocimiento y la forma de aprehenderlo.

En primera instancia la guía Swebok permite tener un referente del contenido temático sugerido para las áreas de conocimiento que componen la Ingeniería del software, en este estudio se abordan las áreas Requisitos de Software y Gestión de la Ingeniería del Software desglosadas en la Tabla 1. 
Tabla 1. Contenidos áreas Requisitos de Software y Gestión de la Ingeniería del Software según Swebok

\begin{tabular}{|l|l|}
\hline Requisitos de software & Gestión de la ingeniería de software \\
\hline Requisitos Fundamentales de Software & Definición de la Iniciación y alcance \\
\hline Procesos de requisitos & Planificación del proyecto software \\
\hline Elicitación de requisitos & Promulgación del proyecto software \\
\hline Análisis de Requisitos & Revisión y evaluación \\
\hline Especificación de Requisitos & Cierre \\
\hline Validación de Requisitos & Medición de la Ingeniería de Software \\
\hline Consideraciones prácticas & $\begin{array}{l}\text { Herramientas de gestión de la ingeniería de } \\
\text { software }\end{array}$ \\
\hline Herramientas para requisitos de software & --- \\
\hline
\end{tabular}

\section{Enfoque de las asignaturas en la Institución}

De acuerdo al Proyecto Académico Educativo (PAE) del programa de Ingeniería de Sistemas y Computación (EISC) de la UPTC, distribuido en 10 semestres, ubica las áreas de este caso de estudio Ingeniería de Requisitos y Gerencia Informática en quinto y noveno semestre respectivamente; para las cuales define los contenidos mínimos programáticos inherentes (Ver Tabla 2).

Tabla 2. Grandes Ejes Temáticos Asignaturas Ingeniería de Requisitos y Gerencia Informática EISC -UPTC

\begin{tabular}{|l|l|}
\hline Ingeniería de Requisitos & Gerencia Informática \\
\hline Ingeniería del software & Fases de la gerencia de proyectos \\
\hline $\begin{array}{l}\text { Actividades y generalidades de la Ingeniería de } \\
\text { Requisitos }\end{array}$ & $\begin{array}{l}\text { Proyectos informáticos e Industria del } \\
\text { software }\end{array}$ \\
\hline $\begin{array}{l}\text { Técnicas y herramientas en la Ingeniería de } \\
\text { Requisitos }\end{array}$ & Gestión de proyectos de software \\
\hline Estándares y especificación de requisitos & Gestión de personal \\
\hline Análisis de requisitos & Estimación de costos del software \\
\hline Validación de requisitos & Gestión de calidad y mejora de procesos \\
\hline Consideraciones prácticas & Gestión de la configuración \\
\hline & Evaluación de proyectos software \\
\hline & Otros tópicos de la gerencia informática \\
\hline
\end{tabular}

\section{Estrategias Pedagógicas en la enseñanza de la Ingeniería de Software}

Para identificar el estado actual de las estrategias pedagógicas usadas en la EISC, fue necesaria la recolección de información de todas aquellas tácticas que aplicadas a las temáticas de las áreas analizadas, permitan la transmisión adecuada del conocimiento, para esto se realiza una selección de estrategias de acuerdo a los niveles de la Taxonomía de Bloom, mostrados en la figura 1, además de elementos pedagógicos reafirmados por diferentes autores y actores del proceso, (Opinion y McLain, 2012; Anderson y Krathwohl, 2002; Harris y Johnson, 2004), los cuales proponen a su juicio tipos de estrategias que pueden ser aplicadas para cada uno de estos niveles, y convertirlos en un instrumento fundamental para el docente Universitario de acuerdo a su perfil de enseñanza (Marín y Conchado, 2012). Dichas estrategias fueron tenidas en cuenta para la presentación de una propuesta en la UPTC, con la que se busca salir de la tendencia de los cursos tradicionales universitarios en los que se entrega información de la misma manera a todos los estudiantes, sin tener en cuenta las diversas estrategias y tipos de aprendizaje. (Huapaya et al., 2005). 


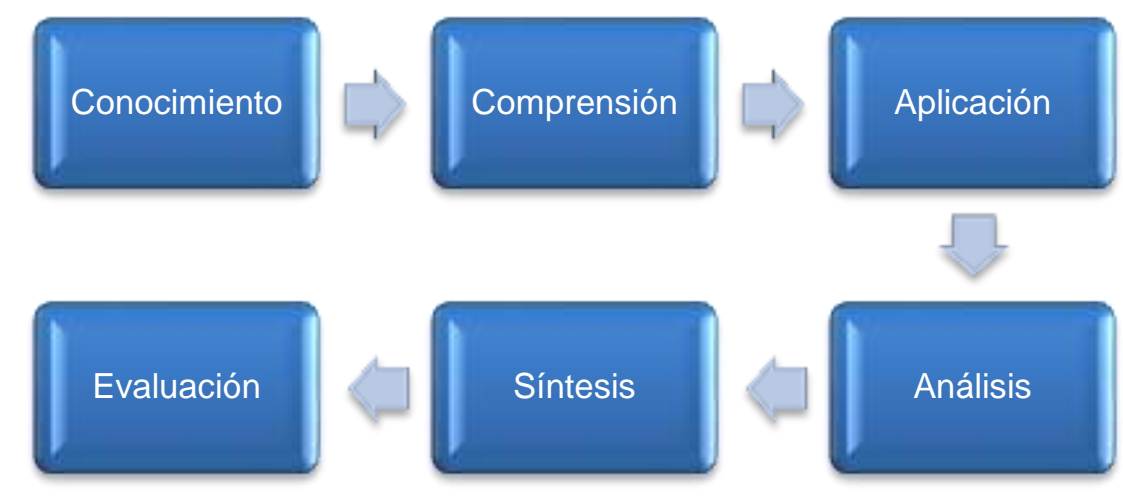

Fig. 1. Dominio cognoscitivo de la Taxonomía de Bloom (Adaptado de Abran et al., 2004)

\section{Identificación de estrategias utilizadas en la EISC}

Con el propósito de obtener la información precisa del estado actual de las áreas de estudio en el ámbito académico de la EISC, se diseña y aplica encuestas diagnosticas tanto a los docentes del área, como a los estudiantes. La encuesta está estructurada así: Presentación, revisión de temáticas de acuerdo a Swebok, medición de la importancia y nivel de aprendizaje de las temáticas y finalmente la revisión de estrategias aplicadas, componentes que se describen a continuación.

Presentación: Incluye una breve introduccion y explicación del objetivo de la encuesta, las áreas que se van a abordar y una estimación del tiempo que toma diligenciar la encuesta. Además se le adicionan opciones para identificar el estado actual de los encuestados, es decir, si son: Estudiantes de últimos semestres, estudiantes con terminación académica, o egresados.

Revisíon de temáticas (Swebok): En este componente de la encuesta se presentan las temáticas que provee Swebok, relacionadas con las áreas inherentes a la investigación, en las cuales el encuestado selecciona las temáticas abordadas en alguna de las asignaturas, y en qué modalidad: Teórica, Práctica o Teórica-Práctica.

Medición de Importancia y Nivel de Aprendizaje de las temáticas planteadas: En esta sección se incluye dos preguntas que permiten evaluar de 1 a 5 el nivel de importancia que tiene para el encuestado esa temática específica, y el nivel de aprendizaje que considera fue obtenido en el proceso de aprendizaje en el aula.

Revision de Estrategias Pedagógicas Aplicadas: En esta sección el encuestado selecciona la asignatura en la que abordó las temáticas relacionadas con las áreas de estudio, y correlaciona las estrategias pedagógicas aplicadas en cada una de éstas.

\section{Fase II. Análisis y selección de información}

Esta fase se orienta al análisis de los documentos existentes para la estructuración de contenidos en ingeniería de requisitos y gerencia informática (mapeo de asignaturas), también el análisis de los datos obtenidos a partir de la aplicación de las encuestas y el análisis del rendimiento académico a partir del historial de calificaciones, para identificar la pertinencia en cuanto a contenidos temáticos y estrategias pedagógicas utilizadas en las mencionadas asignaturas.

\section{Mapeo de Asignaturas}

Se identifica la correlación entre las temáticas presentadas por la guía y las contempladas por los contenidos de cada asignatura para determinar cuales se encuentran dentro del plan de estudios descrito por el PAE y en qué asignatura se encuentran incluidas, y/o cuales de las propuestas en la Guía Swebok no son contempladas en el programa académico. Para realizar el mapeo es importante comprender que la guía Swebok, se divide en áreas de conocimiento (KA por sus siglas en inglés) las cuales abordan una temática general, y estas áreas a su vez se subdividen en temas o sub-áreas, para detallar el contenido de las mismas. De forma análoga, en la EISC se tienen en cuenta las Asignaturas y en el marco de éstas se desarrollan temáticas generales, distribuidas en temas establecidos en el PAE. 


\section{Análisis de Encuestas}

Teniendo en cuenta que para el caso de estudio se aplicó la encuesta a la totalidad de la población, compuesta por los docentes que orientan o han orientado las asignaturas que hacen parte del área de ingeniería de software; la encuesta docente permitió identificar el grado de conocimiento y aplicación de la Guía Swebok en la academia, de acuerdo a la opinión de los docentes del área de Ingeniería del Software, dando como principal resultado que la guía Swebok es conocida por el $75 \%$ del total de encuestados, y que sólo el $25 \%$ del total de docentes encuestados, ha usado la guía como herramienta para la enseñanza de sus asignaturas y que solamente el $33 \%$ de los docentes que tienen conocimiento de la Guía Swebok han estructurado los contenidos de su asignatura (o asignaturas) con base en dicho documento y los aplica en el desarrollo de sus clases, resultados parciales que reafirman la necesidad del uso de esta guía como apoyo para el afianzamiento del aprendizaje.

En el caso de la comunidad estudiantil de la EISC, la población está conformada por los estudiantes de últimos semestres (quienes ya han cursado las dos asignaturas seleccionadas para el estudio) y egresados del programa académico. La percepción estudiantil se orienta a la identificación del cumplimiento de temáticas de las asignaturas frente a los contenidos planteados por Swebok, la metodología, importancia y nivel de aprendizaje asimilado por el estudiante/egresado del programa, de tal manera que el resultado presenta evaluaciones poco satisfactorias debido a que se considera (según los encuestados) que las áreas en estudio tienen alto grado de importancia en la formación profesional, pero las estrategias y cobertura temática presentan elementos que requieren replantearse con el propósito de aprehender y asimilar los conceptos para la cotidianeidad del ingeniero de sistemas; dichos valores obtenidos en las encuestas se convierten en el insumo principal para evaluar el estado actual de contenidos y estrategias pedagógicas usadas actualmente en la EISC frente la guía Swebok.

\section{Análisis académico de las calificaciones obtenidas en las asignaturas caso de estudio.}

Parte importante de la información referente al rendimiento académico fue obtenido de las estadísticas que posee el Sistema de información de Registro Académico (SIRA) de la UPTC, teniendo en cuenta el promedio académico anual, que será tomado como valor del aprendizaje teórico, en el análisis posterior de los mapeos de contenidos, el periodo de análisis fueron los años 2005 al 2013.

\section{Fase III. Evaluación de asignaturas}

Una de las evaluaciones relevantes en el trabajo es la realizada a las asignaturas, tomando como base el modelo de Gradación de Juicios de la UPTC, documento oficial de la Institución en el que se establece elementos cualitativos y cuantitativos para medir la calidad de los programas académicos, con base en la asignación del grado de cumplimiento de ciertos requerimientos (en escala de 0.0 a 5.0), y se establece una ponderación a cada elemento evaluado, para que junto al grado de cumplimiento sea posible definir una emisión de juicio de carácter evaluativo (UPTC, 2011), como se observa en la Tabla 3.

Tabla 3. Escala de Calificación

\begin{tabular}{|l|l|}
\hline Calificación & Grado de Cumplimiento \\
\hline $4.7-5.0$ & Pleno \\
\hline $4.0-4.6$ & Alto Grado \\
\hline $3.0-3.9$ & Aceptable \\
\hline $2.0-2.9$ & Insatisfactorio \\
\hline $1.0-1.9$ & Deficiente \\
\hline $0.0-0.9$ & No Cumple \\
\hline
\end{tabular}

\section{Evaluación de características en las asignaturas}

La evaluación de las asignaturas se llevó a cabo utilizando el principio "divide y vencerás" ya que cada una de las asignaturas se dividió en temáticas (teniendo como base la Guía Swebok), dichas temáticas a su vez fueron divididas en características y éstas en indicadores, de tal manera que se individualizó la calificación de indicadores, y su ponderado era la calificación de la característica correspondiente, de igual manera el ponderado de características se asocia a la calificación de la temática, y finalmente el ponderado del conjunto de temáticas es asociado a la calificación final de la asignatura. 


\section{ANÁLISIS DE RESULTADOS}

Para el análisis del proceso se ha tenido en cuenta los factores principales, como son el cumplimiento de temáticas, el uso de estrategias metodológicas y pedagógicas, y la evaluación de dichas estrategias frente al nivel de aprendizaje de las temáticas.

\section{Cumplimiento de Temáticas EISC Vs. Swebok.}

La aplicación del proceso de gradación de juicios asociado a la característica denominada "Cumplimiento de Temáticas" frente a las temáticas propuestas por Swebok, denota que en la asignatura Ingeniería de requisitos no obtiene una satisfactoria evaluación (3.64) como se muestra en la tabla 4, la principal razón es debido a que su modalidad es práctica y las actividades desarrolladas no conllevan al uso de conceptos y teorías en un ambiente experimental.

Tabla 4. Evaluación del Cumplimiento de Temáticas en Ingeniería de Requisitos frente a Swebok

\begin{tabular}{|l|c|}
\hline \multicolumn{1}{|c|}{ Temática } & Ingeniería de Requisitos \\
\hline Requisitos Fundamentales de Software & 3.73 \\
\hline Procesos de requisitos & 3.83 \\
\hline Elicitación de requisitos & 4.24 \\
\hline Análisis de Requisitos & 3.27 \\
\hline Especificación de Requisitos & 4.51 \\
\hline Validación de Requisitos & 3.24 \\
\hline Consideraciones prácticas & 2.67 \\
\hline \multicolumn{2}{|c|}{ Calificación } \\
\hline
\end{tabular}

De la misma manera en la asignatura Gerencia Informática se obtiene una calificación de cumplimiento de temáticas respecto a Swebok de 2.45 (Insatisfactoria), esto debido, entre otros, a que el factor "Revisión y Evaluación" y "Cierre", según el nivel de taxonomía que requiere es de aplicación, y esta asignatura está contemplada en modalidad Teórica, dichos resultados se sintetizan en la Tabla 5.

Tabla 5. Evaluación del Cumplimiento de Temáticas en Gerencia Informática frente a Swebok

\begin{tabular}{|l|c|}
\hline \multicolumn{1}{|c|}{ Temática } & Gerencia Informática \\
\hline Definición de la Iniciación y alcance & 2.7 \\
\hline Planificación del proyecto software & 3.32 \\
\hline Promulgación del proyecto software & 2.72 \\
\hline Revisión y evaluación & 1.58 \\
\hline Cierre & 1.38 \\
\hline Medición de la Ingeniería de Software & 3.02 \\
\hline \multicolumn{2}{|c|}{ Calificación } \\
\hline \multicolumn{2}{|c|}{2.45} \\
\hline
\end{tabular}

En los dos casos se encontró que aunque se aborda los principales elementos teóricos, aún se quedan otros sin incluir en el contenido temático, que (por tiempo en la mayoría de los casos) se orientan únicamente de forma teórica dejando elementos prácticos para que el estudiante experimente por su propia cuenta. Es importante mencionar que los temas asociados a las herramientas de cada área (incluidas en la versión 3 de 2014), no fueron evaluados en este trabajo, ya que en el momento de la aplicación de los instrumentos se encontraba vigente la versión de Swebok 2004.

\section{Pertinencia en las estrategias metodológicas utilizadas en la EISC}

Con los datos obtenidos en los análisis de encuestas (docente y estudiantil), mapeos, y revisiones documentales, se detecta que para la enseñanza de la Asignatura Ingeniería de Requisitos como se muestra en la figura 2, las estrategias más usadas son las que corresponden a modalidades teóricas, 
destacando: exposiciones, debates, método de proyectos y panel de discusión; en el mismo contexto, para la asignatura Gerencia Informática, en la figura se observa que las estrategias más usadas son las que corresponden a modalidades teóricas, entre estas: debates, panel de discusión, exposiciones y sistema de instrucción personalizada. Información que permite deducir que las estrategias pedagógicas y metodológicas usadas actualmente para la enseñanza de las asignaturas Ingeniería de Requisitos y Gerencia Informática, están orientadas a aspectos teóricos, lo cual es un aspecto que podría mejorarse teniendo en cuenta los niveles de taxonomía de Bloom, involucrando otras estrategias que permitirían una mejor aprehensión del conocimiento en estas áreas.

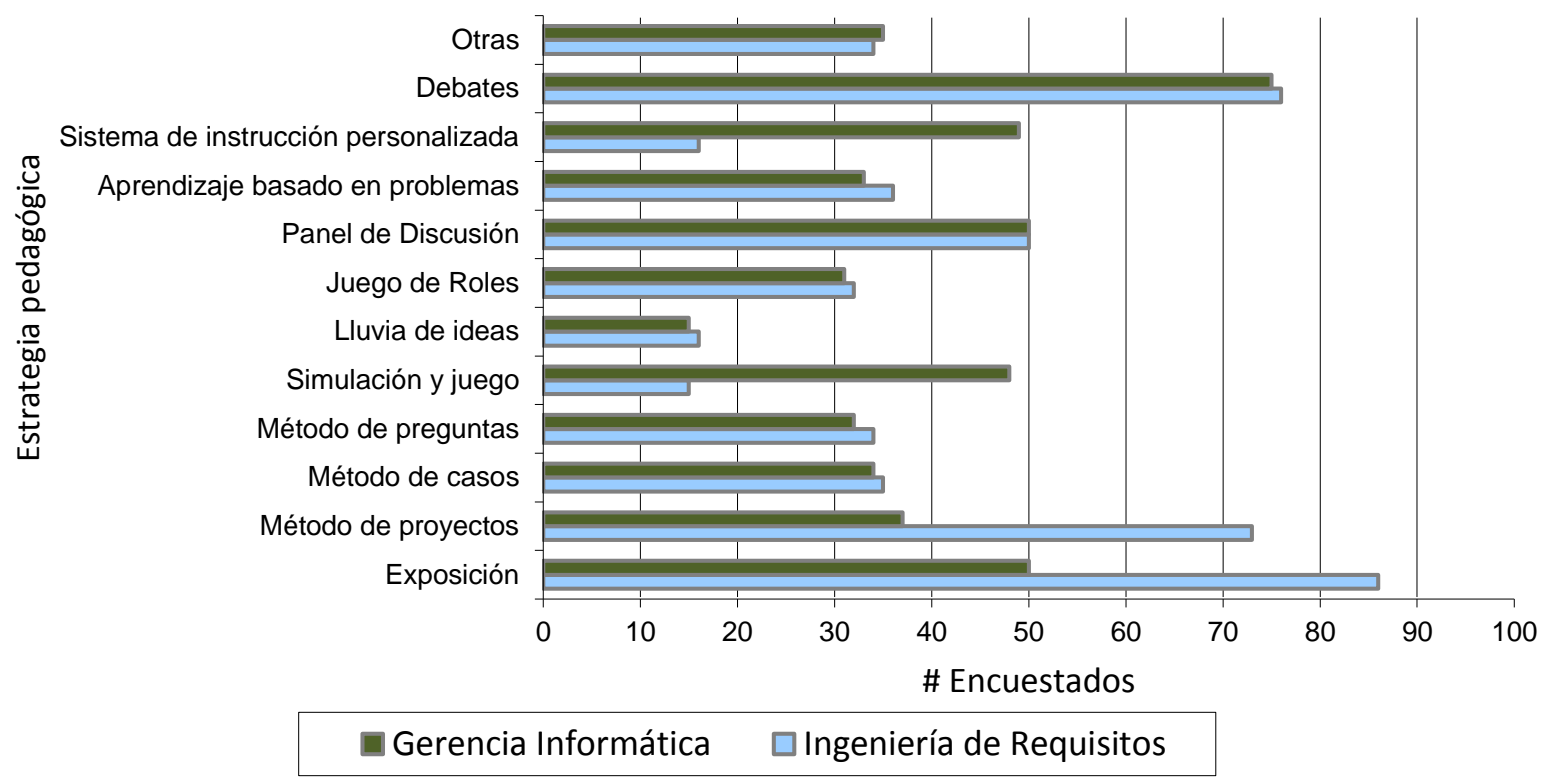

Fig. 2. Estrategias Usadas en Ingeniería de Requisitos y Gerencia Informática

\section{Evaluación de estrategias pedagógicas frente al nivel de aprendizaje}

Teniendo en cuenta el análisis de estrategias utilizadas en la EISC y su correlación con el rendimiento académico en las asignaturas caso de estudio (figura 3), es posible identificar que en el promedio acumulado anualmente predomina un comportamiento "satisfactorio" (calificación entre 3.5 y 3.9 según escala de evaluación de la UPTC), inducido en la mayoría de casos por las estrategias teóricas utilizadas, pero es de mencionar que según la percepción de los estudiantes y egresados la práctica de dichos conceptos y teorías hubiera sido un excelente complemento para la asimilación de las temáticas y la integralidad de su formación profesional. Se tiene en cuenta que la asignatura Gerencia Informática se orienta en el programa sólo a partir del año 2008.

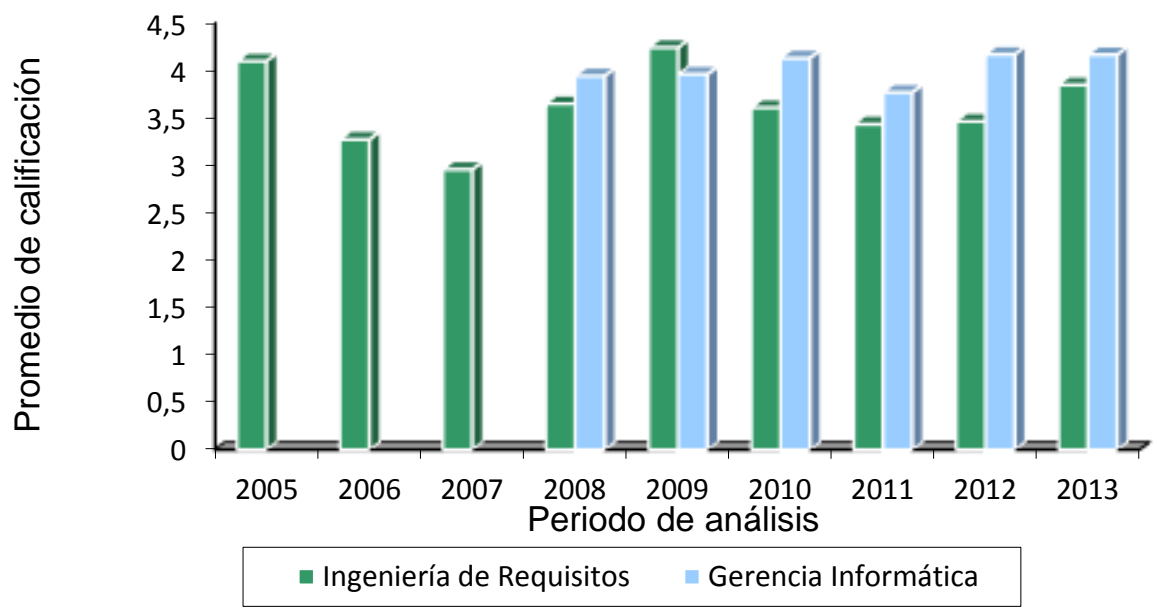

Fig. 3. Rendimiento académico expresado en el promedio de calificaciones del periodo 2005-2013 


\section{DESCRIPCIÓN DE LA PROPUESTA}

A partir de los resultados de la investigación, y teniendo en cuenta las teorías educativas, principalmente la de Louis Not (Not, 1994) y el estudio de viabilidad presentado en el artículo Use of Learning Strategies of Swebok@ guide Proposed Knowledge Areas" (Alarcón et al., 2013), se propone un modelo de transferencia del conocimiento preciso y aplicable al mejoramiento del aprendizaje-enseñanza de las áreas de estudio afín al modelo establecido dentro de la EISC (ver Figura 4).

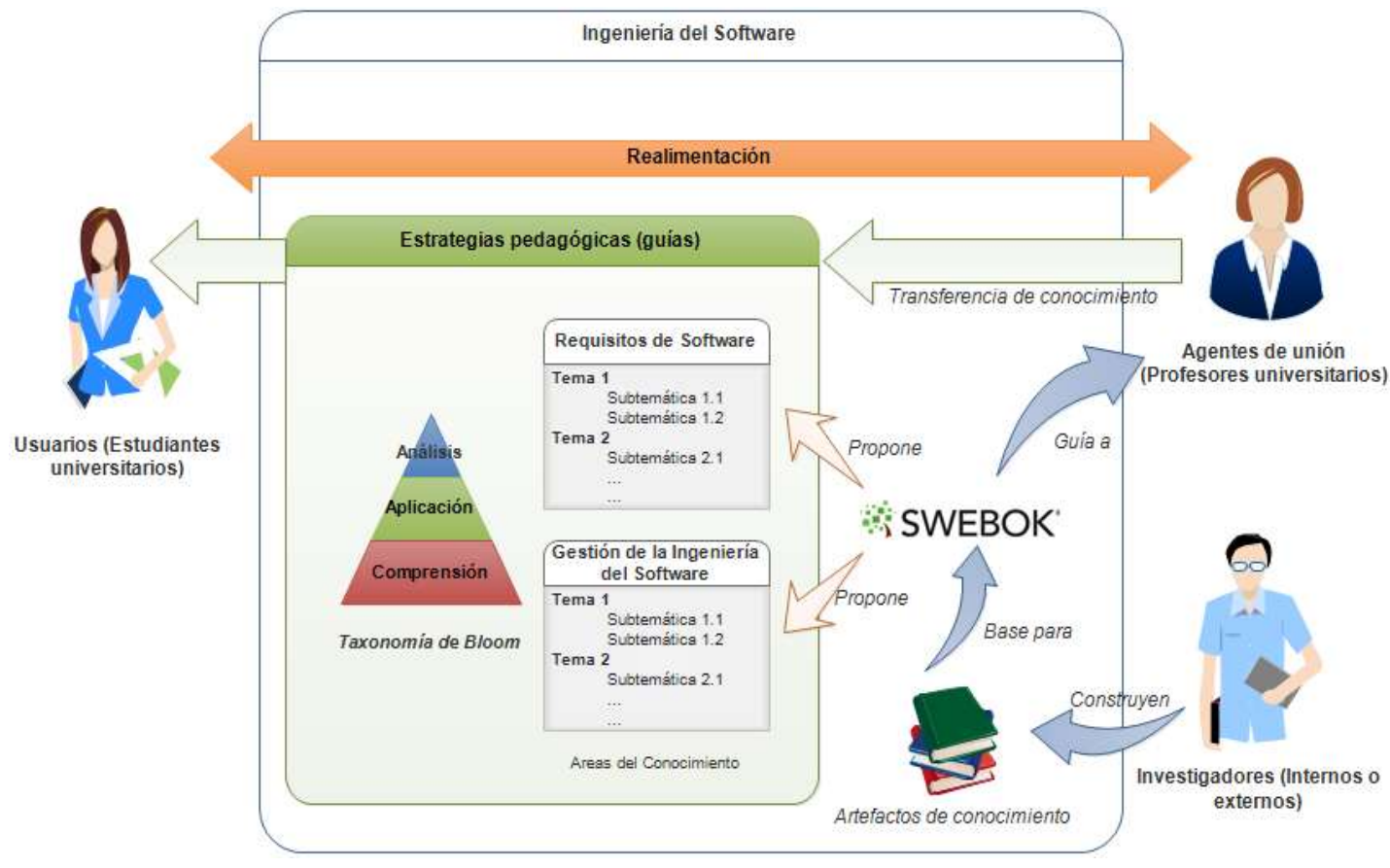

Fig. 4. Modelo pedagógico propuesto

Dicho modelo se compone de los actores descritos a continuación, de tal forma que pueden llegar a optimizar la enseñanza-aprendizaje de la Ingeniería de Requisitos y Gestión de la Ingeniería de Software:

Guía Swebok. Se compone de diferentes artefactos de conocimiento construidos por diversos tipos de investigadores. Propone áreas de conocimiento específicas que componen la Ingeniería del Software.

Estrategias Pedagógicas (Guías). Son construidas para la enseñanza de las áreas Requisitos de Software y Gestión de la Ingeniería del Software propuestas por Swebok, y toman como base la Taxonomía de Bloom para enfocarlas de manera tal que se desarrollen las competencias de los estudiantes más eficazmente y se optimice el aprendizaje.

Agentes de Unión. Son los docentes o profesores universitarios que usarán Swebok para construir las estrategias como guía para la preparación de sus clases. Estos serán los encargados de transferir el conocimiento a los Usuarios por medio de la aplicación de las estrategias

Usuarios. Son los estudiantes, quienes desarrollarán las guías de aprendizaje-enseñanza y a los cuales se les aplicara la estrategia indicada para la temática que se esté evaluando. También y como afianzamiento del conocimiento deben realimentar con los Agentes de Unión sus experiencias y las incidencias presentadas durante el aprendizaje.

Investigadores. Son las personas que están al tanto de los nuevos avances y cambios que se presentan en la ingeniería del software, y son los encargados de la producción científica de la Guía Swebok.

\section{CONCLUSIONES}

Las áreas Ingeniería de Requisitos y Gerencia Informática utilizan en mayor medida las estrategias que aplican más al nivel de Comprensión de taxonomía de Bloom (teórico), dejando en un segundo plano la práctica de contenidos, lo que conlleva a niveles medio - bajo de aprehensión del conocimiento, ya sea por el uso inapropiado de las estrategias pedagógicas, o por la ausencia de actividades prácticas. 
La evaluación de las asignaturas denota que el área Ingeniería de requisitos evaluada con 3.64 correspondiente a un $72.8 \%$ de cumplimiento de contenidos frente a la guía Swebok, y en este aspecto la asignatura Gerencia Informática con una evaluación de 2.45 , cumple en un $49 \%$, lo que indica que es necesario revisar los contenidos programáticos, y poder abarcar en mayor proporción las temáticas propuestas por la guía Swebok, para alcanzar un mejor desempeño docente y un mejor apropiación del conocimiento por parte de los estudiantes lo que redundará en mejores competencias profesionales.

A partir del análisis diagnóstico realizado en la EISC de la Universidad, en las áreas de Ingeniería de Requisitos y Gerencia Informática, y teniendo como base principios pedagógicos, es posible determinar una estrategia que se adapte a las características propias de la Ingeniería de Software, con el propósito de propender por el mejoramiento del rendimiento académico en dicha área, pero también en pro de la asimilación y aprehensión de los conceptos conducentes a contar con profesionales mejor capacitados para ofrecer soluciones tecnológicas afines a las necesidades reales de la sociedad, para lo cual la propuesta descrita debe ser implementada y refinar cada uno de sus componentes.

\section{REFERENCIAS}

Abran, A., P. Bourque y L. Tripp, "Guide to the Software Engineering Body of Knowledge”, (En línea), http://www.math.unipd.it/ tullio/IS-1/2007/Approfondimenti/Swebok.pdf, Acceso 10 de Abril 2016.

Alarcón, A., A. Martínez y J. Sandoval, “Use of Learning Strategies of Swebok@ Guide Proposed Knowledge Areas", Actas 7th International Conference on Knowledge Management in Organizations, 243-254, Salamanca, España (2013)

Alarifi, A., M. Zarour, N. Alomar, Z. Alshaikh y M. Alsaleh, "SECDEP: Software Engineering Curricula Development and Evaluation Process using Swebok", doi:10.1016/j.infsof.2016.01.013, Inf. Softw. Technol. 74, 114-126 (2016)

Anderson, L. W. y D. R. Krathwohl, "A Taxonomy for Learning, Teaching, and Assessing: A Revision of Bloom's Taxonomy of Educational Objectives", Theory into Practice, 41(4), 210 -267 (2002)

Ardis, M., P. Bourque, T. Hilburn, K. Lasfer, S. Lucero, J. McDonald, A. Pyster y M. Shaw, “Advancing Software Engineering Professional Education”. IEEE Software 28(4), 58-63 (2011)

Bourque, P. y R. Fairley, "Guide to the Software Engineering Body of Knowledge, Version 3.0", IEEE. (En línea), http://www4.ncsu.edu/ tjmenzie/cs510/pdf/SWEBOKv3.pdf, Acceso 10 de Abril 2016.

Harris, P. y R. Johnson, "Teaching learning - Montana State University”. (En línea), http://www.montana.edu/teachlearn/Papers/activelearn2.pdf, Acceso 12 Marzo 2016.

Huapaya, C., G. Arona y F. Lizarralde, "Enseñanza de la Ingeniería con Sistemas Tutoriales Inteligentes”, Información tecnológica, 16(5), 75-78 (2005)

IEEE ISO/IEC/IEEE 29148:2011, "Systems and software engineering - Life cycle processes Requirements engineering”, New York, USA (2011)

Kakeshita, T, "Relationship Analysis among Curriculum, Qualification, BOK and Task Profile in ICT Field", IEEE 3rd International Conference on Innovation and Technology in Education (MITE), 117-122, Amritsar, India, (2015)

Marín, J. y A. Conchado, "Influencia de los Perfiles Docentes en el Uso y Preferencia de Dinámicas de Trabajo en Grupo con Alumnos Universitarios”, Formación Universitaria, 5(5), 3-14 (2012)

Nokes, S.," The Definitive Guide to Project Management”. 2nd edición, Financial Times / Prentice Hall, Londres, Inglaterra (2007)

Not, L., “Las pedagogías del conocimiento”. Fondo de Cultura Económica, Bogotá, Colombia, (1994)

Offutt, J., "SE 2014: Curriculum Guidelines for Undergraduate Degree Programs in Software Engineering", Computer, 48, 31-34 (2015)

Opinion Dynamics Corporation y McLain ID Consulting, "2010-2012 Workforce Education and Training (WE\&T) Process Evaluation Volume I: Centergies”, (En línea), http://www.calmac.org/publications/20102012_WE\%26T_Centergies_Process_Eval_Report_volume_I.pdf, Acceso 5 de Abril 2016. 
Osuna, C. y E. Luna, "Valores Éticos en la Formación Universitaria de las Áreas de Ciencias Naturales e Ingeniería y Tecnología, en el Contexto de la Sociedad del Conocimiento", Formación Universitaria, 4(5), 29$36(2011)$

PMI, "What is Project Management?", (En línea), http://www.pmi.org/About-Us/About-Us-What-is-ProjectManagement.aspx, Acceso 14 Abril 2016.

Quezadaa, P., J. Garbajosa, L. Enciso, L. Solano y L. Barba, "Estructura, Componentes e Importancia de los Cuerpos de Conocimiento en el contexto de la Ingeniería de Software y propuesta de dos nuevas áreas de conocimiento en la currícula de la Titulación de Sistemas Informáticos", Rev. Tecnológica ESPOL-RTE 28, 133-146 (2015)

Ramakrishnan, S, "Accreditation of Monash University Software Engineering (MUSE) Program", Informing Science \& Information Technology, 4, 73-89 (2007)

Samarthyam, G., G. Suryanarayana, A. Gupta y R. Nambiar, "FOCUS: An Adaptation of a Swebok-Based Curriculum for Industry Requirements", International Conference on Software Engineering, 1215-1224, Zurich, Suiza (2012)

Sedelmaier, Y. y D. Landes, "SWEBOS - The Software Engineering Body of Skills", DOI: 10.3991/ijep.v5i1.4047, International Journal of Engineering Pedagogy, 5 (1), 20-26 (2014)

Talib, M, A. Khelifi, y L. Jololian, "Secure Software Engineering: A New Teaching Perspective Based on the Swebok", Interdisciplinary Journal of Information, Knowledge \& Management, 5, 83-99 (2010)

UPTC, "Procedimiento de Autoevaluación de Programas: Taller de Gradación de Cumplimiento y Emisión de Juicios: Universidad Pedagógica y Tecnológica de Colombia", (En línea), http://virtual.uptc.edu.co/acreditacion/MODELO/ANEXOS/GUIASYFORMATOS/GUIAS/GUIA_03_GRADACI ON_JUICIOS.pdf, Acceso 4 de Abril 2016. 